\title{
GEOMETRIC REALIZATION AND K-THEORETIC DECOMPOSITION OF C*-ALGEBRAS
}

\author{
C. L. SCHOCHET \\ Mathematics Department \\ Wayne State University \\ Detroit, MI 48202
}

Submitted November 25, 1999

\begin{abstract}
Suppose that $A$ is a separable $C^{*}$-algebra and that $G_{*}$ is a (graded) subgroup of the $\mathbb{Z} / 2$-graded group $K_{*}(A)$. Then there is a natural short exact sequence$$
0 \rightarrow G_{*} \longrightarrow K_{*}(A) \longrightarrow K_{*}(A) / G_{*} \rightarrow 0
$$

In this note we demonstrate how to geometrically realize this sequence at the level of $C^{*}$-algebras. As a result, we $K K$-theoretically decompose $A$ as

$$
0 \rightarrow A \otimes \mathcal{K} \longrightarrow A_{f} \longrightarrow S A_{t} \rightarrow 0
$$

where $K_{*}\left(A_{t}\right)$ is the torsion subgroup of $K_{*}(A)$ and $K_{*}\left(A_{f}\right)$ is its torsionfree quotient. Then we further decompose $A_{t}$ : it is $K K$-equivalent to $\oplus_{p} A_{p}$ where $K_{*}\left(A_{p}\right)$ is the $p$-primary subgroup of the torsion subgroup of $K_{*}(A)$. We then apply this realization to study the Kasparov group $K^{*}(A)$ and related objects.
\end{abstract}

In Section 1 we produce the basic geometric realization. For any separable $C^{*}$ algebra $A$ and group $G_{*}$ we produce associated $C^{*}$-algebras $A_{s}$ ( $s$ for subgroup) and $A_{q}$ ( $q$ for quotient group) and, most importantly, a short exact sequence of $C^{*}$-algebras

$$
0 \rightarrow A \otimes \mathcal{K} \rightarrow A_{q} \rightarrow S A_{s} \rightarrow 0
$$

whose associated $K_{*}$-long exact sequence is $\left(^{*}\right)$. In the case where $G_{*}$ is the torsion subgroup of $K_{*}(A)$ we use the notation $A_{t}$ ( $t$ for torsion) and $A_{f}$ ( $f$ for torsionfree) respectively. We further decompose $A_{t}$ into its $p$-primary summands $A_{p}$ for each prime $p$.

Section 2 deals with the following question: may calculations of the Kasparov groups $K K_{*}(A, B)$ be reduced down to the four cases $\left(A_{t}, B_{t}\right),\left(A_{t}, B_{f}\right),\left(A_{f}, B_{t}\right)$ and $\left(A_{f}, B_{f}\right)$ ? We show that this is indeed possible in a wide variety of situations. Sections 3 and 4 deal with these special cases.

1991 Mathematics Subject Classification. Primary 46L80, 19K35, 46L85.

Key words and phrases. $K$-theory for $C^{*}$-algebras, geometric realization, Kasparov theory. 
Geometric realization as a general technique was introduced to topological $K$ theory of spaces by M. F. Atiyah [1] in his proof of the Künneth theorem for $K^{*}(X \times Y)$. We adapted the technique [6] to prove the corresponding theorem for the $K$-theory for $C^{*}$-algebras and used it with J. Rosenberg in our proof of the Universal Coefficient Theorem (UCT) [4].

\section{Geometric Realization}

In this section we produce the main geometric realization and we extend the result to give a $p$-primary decomposition for a $C^{*}$-algebra.

Let $\mathcal{N}$ denote the bootstrap category $[6,4]$.

Theorem 1.1. Suppose that $A$ is a separable $C^{*}$-algebra. Let $G_{*}$ be some subgroup of $K_{*}(A)$. Then there is an associated $C^{*}$-algebra $A_{s} \in \mathcal{N}$, a separable $C^{*}$-algebra $A_{q}$, and a short exact sequence

$$
0 \rightarrow A \otimes \mathcal{K} \rightarrow A_{q} \rightarrow S A_{s} \rightarrow 0
$$

whose induced $K$-theory long exact sequence fits into the commuting diagram

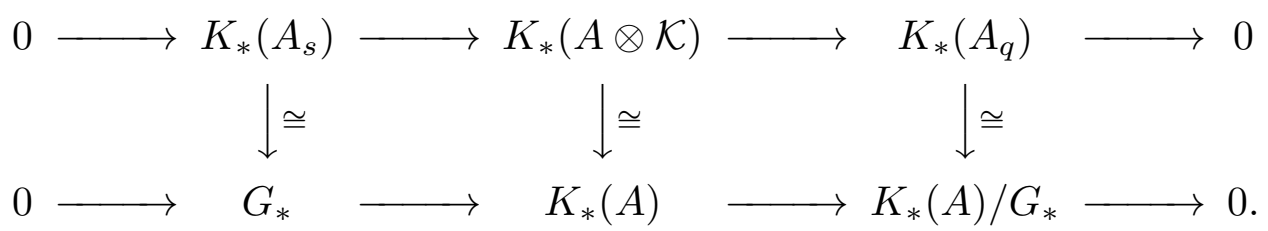

If $A$ is nuclear then so is $A_{q}$. If $A \in \mathcal{N}$ then so is $A_{q}$. If $A \in \mathcal{N}$ and if $G_{*}$ is a direct summand of $K_{*}(A)$ then $A$ is $K K$-equivalent to $A_{s} \oplus A_{q}$.

Note that we think of $A_{s}$ as realizing the subgroup $G_{*}$ and $A_{q}$ as realizing the quotient group $K_{*}(A) / G_{*}$, hence the notation.

Proof. Let $A_{s}$ denote any $C^{*}$-algebra in $\mathcal{N}$ with

$$
K_{*}\left(A_{s}\right) \cong G_{*}
$$

Such $C^{*}$-algebras exist and are unique up to $K K$-equivalence by the UCT [4]. Let

$$
\theta: K_{*}\left(A_{s}\right) \rightarrow K_{*}(A)
$$

be the corresponding homomorphism. Since $A_{s} \in \mathcal{N}$, the UCT holds for the pair $\left(A_{s}, A\right)$, and so $\theta$ is in the image of the index map

$$
\gamma: K K_{*}\left(A_{s}, A\right) \rightarrow \operatorname{Hom}_{\mathbb{Z}}\left(K_{*}\left(A_{s}\right), K_{*}(A)\right) .
$$

Say that

$$
\theta=\gamma(\tau)
$$

for some

$$
\tau \in K K_{0}\left(A_{s}, A\right)
$$

As $A_{s}$ is nuclear, 
and hence $\tau$ corresponds to an equivalence class of extensions of $C^{*}$-algebras of the form

$$
0 \rightarrow A \otimes \mathcal{K} \rightarrow E \rightarrow S A_{s} \rightarrow 0 .
$$

Define $A_{q}=E$. (This choice depends upon the choice of $A_{s}$ among its $K K$ equivalence class and the choice of $\tau$ modulo the kernel of $\gamma$ ). Note that $E$ is nuclear/bootstrap if and only if $A$ is nuclear/bootstrap. Then the diagram

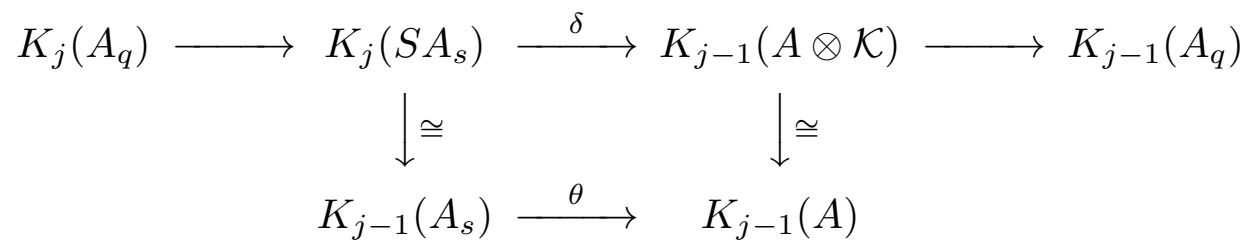

commutes, and thus $\delta$ is mono and the long exact $K_{*}$-sequence breaks apart as shown.

If $G_{*}$ is a direct summand of $K_{*}(A)$ then

$$
K_{*}(A) \cong G_{*} \oplus K_{*}(A) / G_{*} \cong K_{*}\left(A_{s}\right) \oplus K_{*}\left(A_{q}\right) \cong K_{*}\left(A_{s} \oplus A_{q}\right)
$$

and, replacing algebras by their suspensions as needed, the $K K$-equivalence is obtained.

Henceforth we shall regard $A_{s}$ and $A_{q}$ as $C^{*}$-algebras associated to $A$ and $G_{*}$, with the understanding that these are well-defined only up to $K K$-equivalence modulo the kernel of $\gamma$, as explained above.

The next step is to decompose $A_{t}$ into its $p$-primary components.

Theorem 1.4. Let $A \in \mathcal{N}$ and suppose that $K_{*}(A)$ is a torsion group, so that $A=A_{t}$. Then $A$ is $K K$-equivalent to a $C^{*}$-algebra $\oplus A_{p}$, where

$$
K_{*}\left(A_{p}\right) \cong K_{*}(A)_{p}
$$

the p-primary torsion subgroup of $K_{*}(A)$.

Proof. For each prime $p$, choose $N_{(p)} \in \mathcal{N}$ with $K_{1}\left(N_{(p)}\right)=0$ and

$$
K_{0}\left(N_{(p)}\right) \cong \mathbb{Z}_{(p)}
$$

the integers localized at $p$. Define

$$
A_{p}=A_{t} \otimes N_{(p)}
$$

The Künneth formula [6] implies that

$$
K_{*}\left(A_{p}\right) \cong K_{*}\left(A_{t} \otimes N_{(p)}\right) \cong K_{*}\left(A_{t}\right) \otimes K_{*}\left(N_{(p)}\right) \cong K_{*}\left(A_{t}\right) \otimes \mathbb{Z}_{(p)} \cong K_{*}(A)_{p}
$$

as desired. Then

$$
K_{*}\left(\oplus_{p} A_{p}\right) \cong \oplus_{p} K_{*}\left(A_{p}\right) \cong \oplus_{p} K_{*}(A)_{p} \cong K_{*}\left(A_{t}\right)
$$

and another use of the UCT implies that $A_{t}$ is $K K$-equivalent to $\oplus_{p} A_{p}$. 
We summarize:

Theorem 1.5. Suppose that $A$ is a separable $C^{*}$-algebra. Then there is an associated $C^{*}$-algebra $A_{t} \in \mathcal{N}$, a separable $C^{*}$-algebra $A_{f}$, and a short exact sequence

$$
0 \rightarrow A \otimes \mathcal{K} \rightarrow A_{f} \rightarrow S A_{t} \rightarrow 0
$$

whose induced $K$-theory long exact sequence fits into the commuting diagram

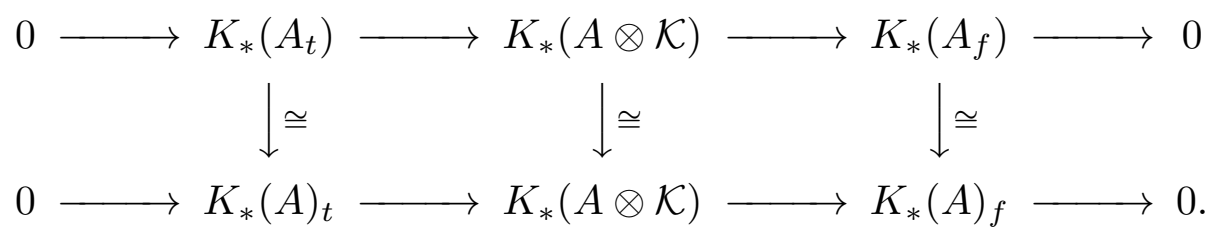

If $A$ is nuclear then so is $A_{f}$. If $A \in \mathcal{N}$ then so is $A_{f}$. Further, the $C^{*}$-algebra $A_{t}$ has a p-primary decomposition: it is $K K$-equivalent to a $C^{*}$-algebra $\oplus_{p} A_{p}$, where $A_{p} \in \mathcal{N}$ for all $p$ and

$$
K_{*}\left(A_{p}\right) \cong K_{*}(A)_{p}
$$

the p-primary torsion subgroup of $K_{*}(A)$. Finally, if $A \in \mathcal{N}$ and $K_{*}(A)_{t}$ is a direct summand of $K_{*}(A)$ then $A$ may be replaced by the $K K$-equivalent $C^{*}$-algebra $A_{t} \oplus A_{f}$

\section{Splitting the Kasparov Groups}

If $A$ and $B$ are in $\mathcal{N}$ and their $K$-theory torsion subgroups $K_{*}(A)_{t}$ and $K_{*}(B)_{t}$ are direct summands then the final conclusion of Theorem 1.5 implies that we may reduce the computation of $K K_{*}(A, B)$ to the calculation of the four groups, namely

(1) $K K_{*}\left(A_{t}, B_{t}\right)$

(2) $K K_{*}\left(A_{t}, B_{f}\right)$

(3) $K K_{*}\left(A_{f}, B_{t}\right)$

(4) $K K_{*}\left(A_{f}, B_{f}\right)$.

We discuss the calculation of those groups in subsequent sections. In this section we see what can be done without assuming that the torsion subgroups are direct summands.

Theorem 2.1. Suppose that $A \in \mathcal{N}$ and $K_{*}(B)$ is torsionfree. Then there is a short exact sequence

$$
0 \rightarrow K K_{*}\left(A_{f}, B\right) \rightarrow K K_{*}(A, B) \rightarrow K K_{*}\left(A_{t}, B\right) \rightarrow 0
$$

In particular, letting $K^{*}(A)=K K_{*}(A, \mathbb{C}$,$) there is a short exact sequence$

$$
0 \rightarrow K^{*}\left(A_{f}\right) \rightarrow K^{*}(A) \rightarrow K^{*}\left(A_{t}\right) \rightarrow 0
$$

If $K_{*}(B)$ is not necessarily torsionfree, then sequence 2.2 is exact if and only if the 


$$
\theta_{h}^{*}: \operatorname{Hom}_{\mathbb{Z}}\left(K_{*}(A), K_{*}(B)\right) \rightarrow \operatorname{Hom}_{\mathbb{Z}}\left(K_{*}\left(A_{t}\right), K_{*}(B)\right)
$$

is onto, where $\theta: K_{*}\left(A_{t}\right) \rightarrow K_{*}(A)$ is the canonical inclusion.

Note that the map $\theta_{h}^{*}$ in (2.4) is frequently onto. This is the case, for instance, if $K_{*}\left(A_{t}\right)$ is a direct summand of $K_{*}(A)$.

The map $\theta$ is, up to isomorphism, the boundary homomorphism in the $K_{*^{-}}$ sequence associated to the short exact sequence

$$
0 \rightarrow A \otimes \mathcal{K} \rightarrow A_{f} \rightarrow S A_{t} \rightarrow 0
$$

and hence

$$
\theta(x)=x \otimes_{A_{t}} \delta
$$

where $\delta \in K K_{1}\left(A_{t}, A\right)$ by [9]. Thus the map $\theta_{h}^{*}$ of (2.4) is induced from a $K K$ pairing.

Proof. Consider the commuting diagram

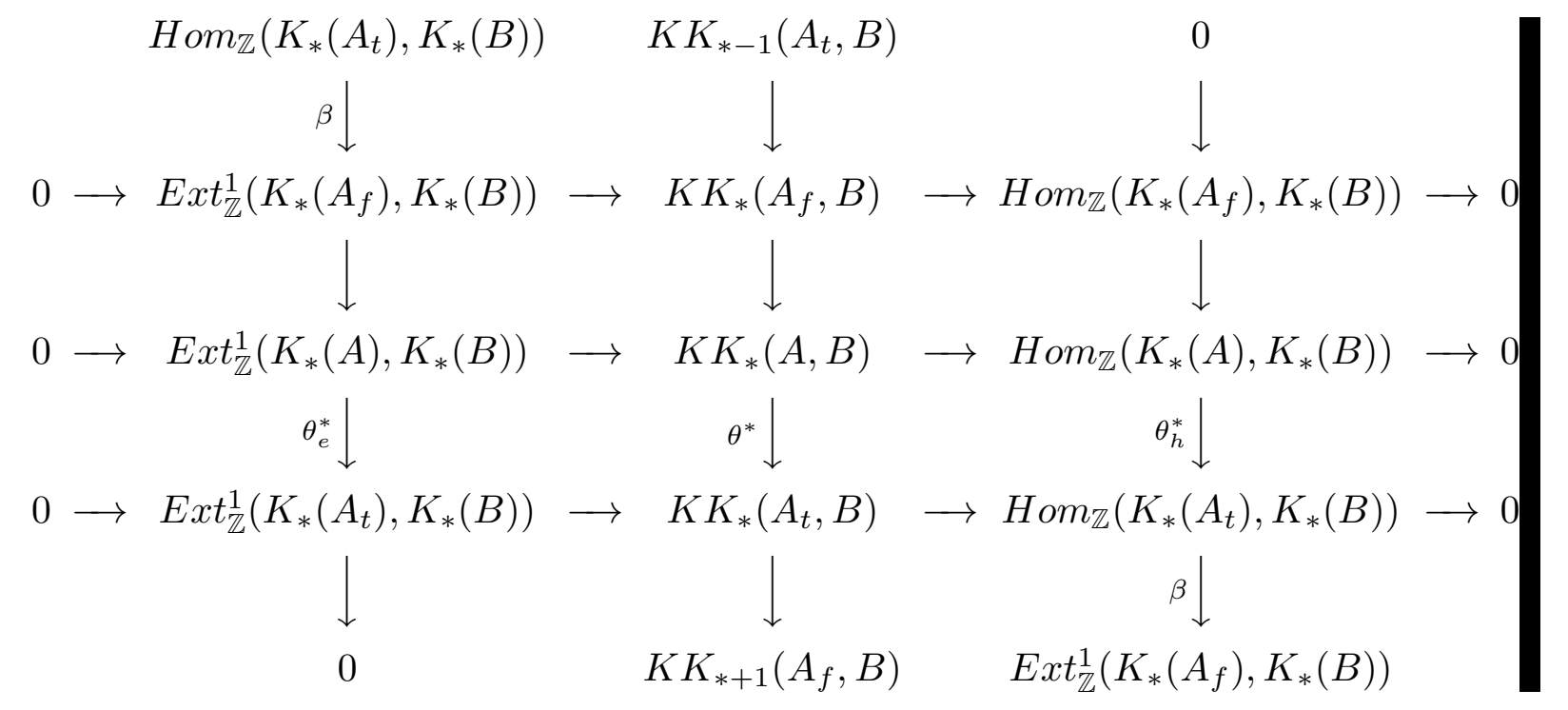

The three middle rows are exact by the UCT, the middle column is exact by the exactness of $K K$, and the two outer columns are exact by the standard Hom-Extsequence.

Suppose that $K_{*}(B)$ is torsionfree. Then

$$
\operatorname{Hom}_{\mathbb{Z}}\left(K_{*}\left(A_{t}\right), K_{*}(B)\right)=0
$$

since $K_{*}\left(A_{t}\right)$ is a torsion group, and the surjectivity of $\theta_{e}^{*}$ implies the surjectivity of $\theta^{*}$.

If $K_{*}(B)$ is not necessarily torsionfree, then the Snake Lemma [11] implies that there is an exact sequence

$$
0=\operatorname{Coker}\left(\theta_{e}^{*}\right) \longrightarrow \operatorname{Coker}\left(\theta^{*}\right) \longrightarrow \operatorname{Coker}\left(\theta_{h}^{*}\right) \rightarrow 0
$$

and hence $\theta_{h}^{*}$ is onto if and only if $\theta^{*}$ is onto. The theorem then follows immediately, for the middle column of the diagram degenerates to (2.2) if and only if $\theta^{*}$ is onto. 
Theorem 2.6. Suppose that $A \in \mathcal{N}$ and that $K_{*}(A)$ is a torsion group. Then there is a natural exact sequence

$$
0 \rightarrow K K_{*}\left(A, B_{t}\right) \rightarrow K K_{*}(A, B) \rightarrow K K_{*}\left(A, B_{f}\right) \rightarrow 0 .
$$

If $K_{*}(A)$ is not a torsion group then sequence $\left({ }^{*}\right)$ is exact if and only if the natural map

$$
\pi_{*}: \operatorname{Hom}_{\mathbb{Z}}\left(K_{*}(A), K_{*}(B)\right) \rightarrow \operatorname{Hom}_{\mathbb{Z}}\left(K_{*}(A), K_{*}\left(B_{f}\right)\right)
$$

is onto, where $\pi: B \otimes \mathcal{K} \rightarrow B_{f}$ is the natural map.

The proof of this result is dual to that of Theorem 2.1 and is omitted for brevity.

\section{Computing $K K_{*}\left(A_{f}, B\right)$}

In this section we consider the case where $K_{*}(A)$ is torsionfree (so that $A=A_{f}$ ). Recall $[2,12]$ that a subgroup $H$ of an abelian group $K$ is pure if for each positive integer $n$,

$$
n H=H \cap n G,
$$

and an extension of groups

$$
0 \rightarrow H \rightarrow K \rightarrow G \rightarrow 0
$$

is pure if $H$ is a pure subgroup of $K$. For abelian groups $G$ and $H, \operatorname{Pext}_{\mathbb{Z}}^{1}(G, H)$ is the subgroup of $\operatorname{Ext}_{\mathbb{Z}}^{1}(G, H)$ consisting of pure extensions.

Recall $[5,8]$ that there is a natural topology on the Kasparov groups and that with respect to this topology the UCT sequence splittings constructed in [4] are continuous, so that the splitting is a splitting of topological groups [9].

Theorem 3.1. Suppose that $A \in \mathcal{N}$ and that $K_{*}(A)$ is torsionfree. Then there is a natural sequence of topological groups

$$
0 \rightarrow \operatorname{Pext}_{\mathbb{Z}}^{1}\left(K_{*}(A), K_{*}(B)\right) \rightarrow K K_{*}(A, B) \rightarrow \operatorname{Hom}_{\mathbb{Z}}\left(K_{*}(A), K_{*}(B)\right) \rightarrow 0
$$

The group $\operatorname{Pext}_{\mathbb{Z}}^{1}\left(K_{*}(A), K_{*}(B)\right)$ is the closure of zero in the natural topology on the group $K K_{*}(A, B)$ and thus the group $\operatorname{Hom}_{\mathbb{Z}}\left(K_{*}(A), K_{*}(B)\right)$ is the Hausdorff quotient of $K K_{*}(A, B)$.

Proof. The UCT gives us the sequence

$$
0 \rightarrow \operatorname{Ext}_{\mathbb{Z}}^{1}\left(K_{*}(A), K_{*}(B)\right) \rightarrow K K_{*}(A, B) \rightarrow \operatorname{Hom}_{\mathbb{Z}}\left(K_{*}(A), K_{*}(B)\right) \rightarrow 0
$$

which splits unnaturally. If $K_{*}(A)$ is torsionfree then

$$
\operatorname{Pext}_{\mathbb{Z}}^{1}\left(K_{*}(A), K_{*}(B)\right) \cong \operatorname{Ext}_{\mathbb{Z}}^{1}\left(K_{*}(A), K_{*}(B)\right) .
$$

The remaining part of the theorem holds since we have shown in general [10] that the group $\operatorname{Pext}_{\mathbb{Z}}^{1}\left(K_{*}(A), K_{*}(B)\right)$ is the closure of zero in the natural topology on $K K_{*}(A, B)$ in the presence of the UCT.

We note that the resulting algebraic problems are frequently very difficult. If $G$ is a torsionfree abelian group then $\operatorname{Hom}_{\mathbb{Z}}(G, H)$ is unknown in general, though there is much known in special cases (cf. [2,3]). The group $\operatorname{Pext}_{\mathbb{Z}}^{1}(G, H)$ is also difficult, though the case $\operatorname{Pext}_{\mathbb{Z}}^{1}(G, \mathbb{Z})$ is known (cf. [3]). We discuss Pext in detail in [12] 


\section{Computing $K K_{*}\left(A_{t}, B\right)$}

In this section we concentrate upon the situation when $K_{*}(A)$ is a torsion group. Before beginning, we digress slightly to recall [7] in more detail how one introduces coefficients into $K$-theory.

Given a countable abelian group $G$, select some $C^{*}$-algebra $N_{G} \in \mathcal{N}$ with

$$
K_{0}\left(N_{G}\right)=G \quad K_{1}\left(N_{G}\right)=0 .
$$

The $C^{*}$-algebra $N_{G}$ is unique up to $K K$-equivalence, by the UCT. Then for any $C^{*}$-algebra $A$, define

$$
K_{j}(A ; G) \cong K_{j}\left(A \otimes N_{G}\right) .
$$

The Künneth Theorem [6] implies that there is a natural short exact sequence

$$
0 \rightarrow K_{j}(A) \otimes G \stackrel{\alpha}{\longrightarrow} K_{j}(A ; G) \rightarrow \operatorname{Tor}_{1}^{\mathbb{Z}}\left(K_{j-1}(A), G\right) \rightarrow 0
$$

which splits unnaturally. If $G$ is torsionfree then $\alpha$ is an isomorphism

$$
\alpha: K_{j}(A) \otimes G \stackrel{\cong}{\longrightarrow} K_{j}(A ; G) .
$$

Let $\mathbf{X}(G)=\operatorname{Hom}(G, \mathbb{R} / \mathbb{Z})$ denote the Pontryagin dual of the group $G$.

Theorem 4.3. Suppose that $A \in \mathcal{N}$ with $K_{*}(A)$ a torsion group and suppose that $K_{*}(B)$ is torsionfree, so that $A=A_{t}$ and $B=B_{f}$. Then:

(3) The group $K K_{*}(A, B)$ is reduced and algebraically compact.

$$
K^{j}(A) \cong \mathbf{X}\left(K_{j-1}(A)\right)
$$

(5) More generally, if $K_{*}(B)$ is finitely generated free, then

$$
K K_{j}(A, B) \cong \oplus_{n} \mathbf{X}\left(K_{j-1}(A)\right)
$$

where $n$ is the number of generators of $K_{*}(B)$.

Proof. Part 1) follows at once from the UCT and the fact that there are no nontrivial homomorphisms from a torsion group to a torsionfree group. Part 2) follows from Part 1) by elementary homological algebra. Part 3) follows easily from a deep result of Fuchs and Harrison [cf. 2, 46.1]: if $G$ is a torsion group then any group of the form $\operatorname{Hom}_{\mathbb{Z}}(G, H)$ is reduced and algebraically compact. Part 4) follows from part 3) by setting $B=\mathbb{C}$ and observing that for any torsion group $G$, we have

$$
\mathbf{X}(G)=\operatorname{Hom}_{\mathbb{Z}}(G, \mathbb{Q} / \mathbb{Z} \text {. })
$$

There is one additional case that fits into the present discussion and which 
Theorem 4.4. Suppose that $A \in \mathcal{N}$ and that $K_{*}(A)$ has no free direct summand. Then there is a natural short exact sequence of topological groups

$$
0 \rightarrow \operatorname{Hom}_{\mathbb{Z}}\left(K_{*}(A), \mathbb{R}\right) \rightarrow \mathbf{X}\left(K_{*}(A)\right) \stackrel{\chi}{\longrightarrow} K^{*}(A) \rightarrow 0 .
$$

The map $\chi: \mathbf{X}\left(K_{*}(A)\right) \rightarrow K^{*}(A)$ is a degree one continuous open surjection. It is a homeomorphism if and only if $K_{*}(A)$ is a torsion group.

To be explicit about the grading,

$$
\chi: \mathbf{X}\left(K_{j}(A)\right) \rightarrow K^{j-1}(A)
$$

which is the usual parity shift as torsion phenomena move from homology to cohomology.

Proof. The UCT for $K^{*}(A)$ has the form

$$
0 \rightarrow \operatorname{Ext}_{\mathbb{Z}}^{1}\left(K_{*}(A), \mathbb{Z}\right) \stackrel{\delta}{\rightarrow} K^{*}(A) \rightarrow \operatorname{Hom}_{\mathbb{Z}}\left(K_{*}(A), \mathbb{Z}\right) \rightarrow 0
$$

with $\delta$ of degree one, so it suffices to compute Ext. In general the short exact sequence

$$
0 \rightarrow \mathbb{Z} \rightarrow \mathbb{R} \rightarrow \mathbb{R} / \mathbb{Z} \rightarrow 0
$$

yields a long exact sequence

$$
\operatorname{Hom}_{\mathbb{Z}}\left(K_{*}(A), \mathbb{Z}\right) \rightarrow \operatorname{Hom}_{\mathbb{Z}}\left(K_{*}(A), \mathbb{R}\right) \rightarrow \mathbf{X}\left(K_{*}(A)\right) \rightarrow \operatorname{Ext}_{\mathbb{Z}}^{1}\left(K_{*}(A), \mathbb{Z}\right) \rightarrow 0 .
$$

The fact that $K_{*}(A)$ has no free direct summand implies that $\operatorname{Hom}_{\mathbb{Z}}\left(K_{*}(A), \mathbb{Z}\right)=0$, so the sequence degenerates to

$$
0 \rightarrow \operatorname{Hom}_{\mathbb{Z}}\left(K_{*}(A), \mathbb{R}\right) \rightarrow \mathbf{X}\left(K_{*}(A)\right) \rightarrow \operatorname{Ext}_{\mathbb{Z}}^{1}\left(K_{*}(A), \mathbb{Z}\right) \rightarrow 0
$$

Applying the UCT one obtains the sequence 4.5 as desired. The map $\chi$ is the composite of the UCT map and a natural homeomorphism. The rest of the Theorem is immediate. 


\section{REFERENCES}

[1] M. F. Atiyah, Vector bundles and the Künneth formula, Topology 1 (1962), 245-248.

[2] László Fuchs, Infinite Abelian Groups, Pure and Applied Mathematics No. 36 , vol. 1, Academic Press, New York, 1970, pp. 290.

[3] C. U. Jensen, Les Foncteurs Dérivés de lim et leur Applications en Théorie des Modules, Lecture Notes in Mathematics, vol. 254, Springer, Verlag, New York, 1972.

[4] J. Rosenberg and C. Schochet, The Künneth theorem and the universal coefficient theorem for Kasparov's generalized K-functor, Duke Math. J. 55 (1987), 431-474.

[5] N. Salinas, Relative quasidiagonality and KK-theory, Houston J. Math. 18 (1992), 97-116.

[6] C. Schochet, Topological methods for $C^{*}$-algebras II: geometric resolutions and the Künneth formula, Pacific J. Math. 98 (1982), 443-458.

[7] C. Schochet, Topological methods for $C^{*}$-algebras IV: $\bmod p$ homology, Pacific J. Math. 114 (1984), 447-468.

[8] C. Schochet, The fine structure of the Kasparov groups I: continuity of the KK-pairing, submitted.

[9] C. Schochet, The fine structure of the Kasparov groups II: topologizing the UCT, submitted.

[10] C. Schochet, The fine structure of the Kasparov groups III: relative quasidiagonality, submitted.

[11 ] C. Schochet, The topological snake lemma and Corona algebras, New York J. Math. 5 (1999), 131-137.

[12] C. Schochet, A Pext Primer: Pure extensions and $\lim ^{1}$ for infinite abelian groups, submitted. 